\title{
A comparison between endoscopic ultrasound-guided rendezvous and percutaneous biliary drainage after failed ERCP for malignant distal biliary obstruction
}

Authors

Institutions
Jason G. Bill', Michael Darcy², Larissa L. Fujii-Lau', Daniel K. Mullady', Srinivas Gaddam¹, Faris M. Murad', Dayna S. Early ${ }^{1}$, Steven A. Edmundowicz ${ }^{1}$, Vladimir M. Kushnir ${ }^{1}$

${ }^{1}$ Division of Gastroenterology, Washington University School of Medicine in St Louis, Missouri, United States

${ }^{2}$ Division of Interventional Radiology, Washington University School of Medicine in St Louis, Missouri, United States submitted

13. February 2016 accepted after revision 5. July 2016

\section{Bibliography}

DOI http://dx.doi.org/

10.1055/s-0042-112584

Published online: 31.8.2016

Endoscopy International Open

2016; 04: E980-E985

(c) Georg Thieme Verlag KG

Stuttgart · New York

E-ISSN 2196-9736

\section{Corresponding author}

\section{Jason G. Bill, MD}

Division of Gastroenterology

Washington University School of Medicine

660 South Euclid Ave

Campus Box 8124

St Louis, Missouri 63110

Phone: +1-314-454-8977

Fax: +1-314-747-1080

jbill@dom.wustl.edu
Background and study Aims: Selective biliary cannulation is unsuccessful in $5 \%$ to $10 \%$ of patients undergoing endoscopic retrograde cholangiopancreatography (ERCP) for malignant distal biliary obstruction (MDBO). Percutaneous biliary drainage (PBD) has been the gold standard, but endoscopic ultrasound guided rendezvous (EUSr) have been increasingly used for biliary decompression in this patient population. Our aim was to compare the initial success rate, long-term efficacy, and safety of PBD and EUSr in relieving MDBO after failed ERC

Patients and methods: A retrospective study involving 50 consecutive patients who had an initial failed ERCP for MDBO. Twenty-five patients undergoing EUSr between 2008-2014 were compared to 25 patients who underwent PBD immediately prior to the introduction of EUSr at our center (2002-2008). Comparisons were made between the two groups with regard to technical success, duration of hospital stay and adverse

\section{Introduction}

$\nabla$

Malignant distal biliary obstruction (MDBO) is a frequent complication of pancreaticobiliary and metastatic malignancy. Endoscopic retrograde cholangiopancreatography (ERCP) is the current gold standard for decompressing the biliary tree in this population [1-4]. However, ERCP can be unsuccessful in $5 \%$ to $10 \%$ of patients with MDBO due to tumor-related alterations in the periampullary anatomy, as well as physician and patient factors [1]. Traditionally, unsuccessful ERCP has been followed by percutaneous biliary drainage (PBD) or surgical intervention to obtain biliary decompression [1]. PBD is a well-established and effective method for biliary decompression in the setting of malignant obstruction. However, in most cases, at least temporary external drainage and repeated interventions for drain maintenance are often required following PBD; leading to po- event rates after biliary decompression.

Results: The mean age at presentation was 66.5 $( \pm 12.6$ years), 28 patients $(54.9 \%)$ were female. The etiology of MDBO was pancreaticobiliary malignancy in $44(88 \%)$ and metastatic disease in $6(12 \%)$ cases. Biliary drainage was technically successful by EUSr in 19 (76\%) cases and by PBD in $25(100 \%)(P=0.002)$. Median length of hospital stay after initial drainage was 1 day in the EUSr group vs 5 days in PBD group $(P=0.02)$. Repeat biliary intervention was required for 4 patients in the EUSr group and 15 in the PBD group $(P=0.001)$.

Conclusions: Initial technical success with EUSr was significantly lower than with PBD, however when EUSr was successful, patients had a significantly shorter post-procedure hospital stay and required fewer follow-up biliary interventions.

Meeting presentations: Annual Digestive Diseases Week 2015

tential treatment interruptions and lower quality of life [5]. Over the past decade, advances in technology and development of new techniques have allowed endoscopic ultrasound guided biliary drainage (EUS-BD) to be used as an alternative in patients with MDBO.

Various techniques of EUS-BD have been described, including EUS-guided choledochoduodenostomy, EUS-guided rendezvous (EUSr), and EUS-guided antegrade stent placement. Early data have demonstrated that in expert hands, EUS-BD is superior to PBD in regards to success of internal biliary drainage and complications and may overall improve a patient's quality of life [4]. The overall technical and clinical success rates of EUS-BD is approximately $80 \%$ with a complication rate of approximately $10 \%$ [6]. Only a few studies have attempted to compare the safety and efficacy of EUS-BD to PBD. In the most recent report, Khashab et al. found that EUS-BD was a 
safer and potentially more efficacious alternative to PBD [2]. However, in this study a variety of EUS-BD methods were employed and PBD was only performed after a failed attempt at EUS-BD.

Because EUS-BD techniques and their role in treating MDBO are still evolving, it remains unclear how outcomes compare to the current gold standard in this setting, PBD. The aim of this study was to compare the initial success rate, long- term efficacy and safety of PBD and EUSr in relieving MDBO after failed initial ERC

\section{Patients and Methods \\ $\nabla$}

This was a retrospective comparative study involving 50 consecutive patients with MDBO in whom biliary decompression could not be achieved via ERCP at a single academic tertiary referral center. Patients were identified from a manual search of the institutional endoscopic database (Provation MD, Minneapolis, MN). A manual chart review was performed and the clinical and endoscopic data were extracted from each patient's electronic medical record including demographics (age, gender), type of malignancy (pancreaticobiliary, metastatic), laboratory data, complications, number of repeat biliary interventions, and length of hospitalization. Twenty-five consecutive patients who underwent EUSr between 2008 and 2014 were compared to a cohort of 25 patients who underwent PBD immediately prior to the introduction of EUSr at our center (2002-2008). All EUSr procedures performed at our institution between 2008 and 2014 were included in this study. Patients with surgically altered anatomy (Roux-En-Y gastric bypass, pancreaticoduodenectomy, hepaticojejunostomy) were excluded from the study. The Institutional Review Board of Washington University School of Medicine/Barnes Jewish Hospital approved review of clinical data for the purpose of this study. EUSr was performed at the university hospital endoscopy unit by 1 of 5 experienced endoscopists expert in EUS, ERCP, and interventional EUS.All patients were under monitored anesthesia care and received antibiotics during the procedure and for 3 to 5 days thereafter. The number of failed ERCP attempts prior to attempting EUSr or PBD was recorded. The definition of failed biliary cannulation with ERCP was not standardized in this study and the decision to use an alternative method of biliary cannulation was determined by the attending endoscopist.

During the time of the study, our group's approach to EUS-BD was to attempt EUSr rather than other techniques after failed ERC All EUSr were performed using a trans-duodenal approach with the echoendoscope seated in the duodenal bulb. Primarily this decision is based on the risk of complications, primarily bile leak, with other EUS-BD methods. After failed ERCP at our center, EUSr was attempted during the same endoscopy session. If EUSr was unsuccessful, the decision to use an alternative method of EUS-BD, refer a patient to PBD, or perform ERCP at a later date was at the discretion of the attending endoscopist. All EUSr procedures were performed using a therapeutic linear echoendoscope (GF-UC140P or GF-UCT140; Olympus Medical Systems, Center Valley, PA). Once the scope was seated in the duodenal bulb, the bile duct was identified and color flow Doppler was used to confirm the lack of intervening vessels. Next, the bile duct was accessed, prior to 2009, using a 19-gauge FNA needle and then using an access needle (EchoTip Access Needle, Cook Medical Inc., Bloomington, IN) thereafter. The needle was advanced into the lumen of the extrahepatic bile duct, the stylet was removed, and bile was aspirated to verify positioning within the bile duct. Next, contrast was injected and a cholangiogram was obtained. Following the cholangiogram, a long guidewire [0.035 inch Jagwire (Boston Scientific Corporation, Natick, MA) or 0.025 inch angled Visiglide wire (Olympus America, Center Valley, PA)] was then passed through the access needle into the bile duct. The guidewire was manually manipulated to advance through the major papilla and was coiled within the duodenum. The access needle and echoendoscope were then removed, leaving the wire in place. Alongside the wire, a duodenoscope (TJF 140 - 160; Olympus Medical Systems, Center Valley, PA) was inserted to the level of the papilla. The biliary tree was then deeply cannulated with a sphinctertome using the guidewire placed via EUSr as a guide to where the biliary opening lay. If cannulation was unsuccessful using this technique, then the luminal end of the guidewire was grasped with a snare or forceps and pulled through the duodenoscope so that both ends of the guidewire exited the patient's mouth and were under the control of the endoscopist. This allowed a sphinctertome or balloon catheter to be advanced over the wire directly into the biliary tree. Subsequently, a biliary stent was placed across the stricture. Technical success of EUSr was defined by the wire being advanced into the duodenum via the major papilla and a stent placed in a retrograde manner ( $\bullet$ Fig. 1 ). Patients were not routinely admitted to the hospital for observation following EUSr if the procedure was performed on an outpatient basis.

PBD was performed by experts in Interventional Radiology under moderate sedation using well established techniques [7]. Most patients initially received external biliary drainage which at the discretion of the attending radiologist could be converted to a metallic biliary stent when clinically appropriate [7]. Patients were monitored in the hospital for a minimum of 23 hours following PBD placement. Following PBD placement, any repeated procedure (stent/tube exchange) was recorded as repeated interventions.

Procedure-related adverse events (AEs) were divided into early (within 7 days) and late (greater than 7 days) from the index procedure. AE recorded included recurrent biliary obstruction, bleeding (defined as an acute $>3 \mathrm{~g} / \mathrm{dL}$ drop in $\mathrm{Hgb}$ requiring PRBC transfusion or overt bleeding outside of what is typically accepted post procedure that required further medical/surgical management), pancreatitis, bile leak and cholecystitis.

\section{Statistical analysis}

Data are reported as mean \pm standard deviation (SD) for normally distributed data, and median and range for skewed data. Grouped continuous variable data were compared using two-tailed Student's t test and Mann-Whitney U test where appropriate. Intergroup and categorical comparisons were made using the Chisquare and Fisher's exact tests. A p value of $<0.05$ was required for statistical significance. All statistical analyses were performed using PASW 19.1 (SPSS, Inc., Chicago, IL).

\section{Results \\ $\nabla$}

During the 12-year study period, 1076 patients underwent an initial ERCP for malignant distal biliary obstruction at our medical center, biliary access via ERCP was unsuccessful in 54 (5.0\%). Four patients elected to pursue hospice care after failed ERCP, and further attempts at biliary decompression were not made. Fifty patients [mean age $66.5 \pm 12.6$ years, 28 (54.9\%) females] were included in this study. The etiology of MDBO was pancreaticobili- 

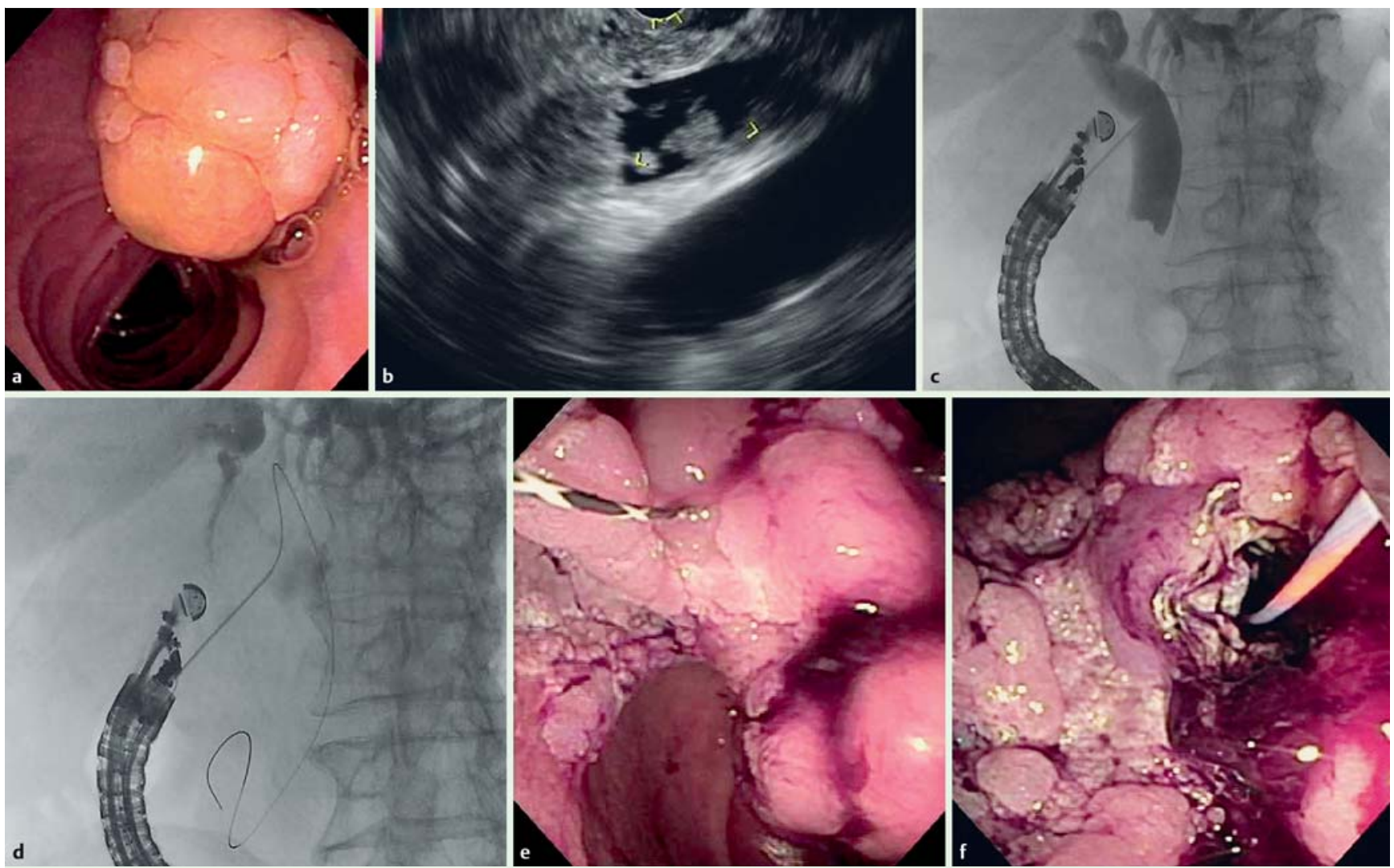

Fig. 1 EUS-guided rendezvous in an 84-year-old patient with obstructive jaundice due to an ampullary cancer. a Endoscopic examination revealed a large mass, which made identification of the biliary orifice impossible. $\mathbf{b}$ Endosonographic examination revealed marked biliary ductal dilation and due to direct extension of the tumor into the bile duct lumen. c Under endosonographic guidance the bile duct was punctured and a cholangiogram was obtained, confirming findings observed on EUS examination. d,e Next, a guide wire was advanced into the duodenum and via the EUS needle. $\mathbf{f}$ Finally a covered metal biliary stent was placed in a retrograde manner.

ary malignancy in 44 (88\%) and metastatic malignancy in $6(12 \%)$ cases. In the EUSr group, pancreaticobiliary malignancies included pancreatic adenocarcinoma in 20 (80\%), neuroendocrine tumor in 1 (4\%), and ampullary/duodenal cancer in 3 (12\%) patients. Patients underwent a median of 1 (range $1-3$ ) unsuccessful ERCP attempts. The reason for failed biliary cannulation at time of ERCP was tumor-related ampullary deformity in 34 (68 $\%$ ) cases and inability to cannulate a normal appearing ampulla in $16(32 \%)$. There were no significant differences in any of the baseline characteristics between the EUSr and PBD groups ( $\bullet$ Table 1$)$.

Biliary drainage was technically successful by EUSr in 19 (76\%) cases and by PBD in 25 (100\%) $(P=0.002$, Table 2$)$. Median length of hospital stay after initial drainage was 1 day (range 0 $26)$ in the EUSr group vs 5 days (range 1-20 days) in the PBD group $(P=0.02)$. The median length of hospital stay following successful EUSr was 1 day (range $0-13$ ), significantly shorter than the PBD group $(P=.002)$. Nine patients in the EUSr group underwent the procedure on an outpatient basis and were not admitted to the hospital within the subsequent 14 days. Clinical success, defined as a $50 \%$ drop in bilirubin within 7 days of the index procedure was obtained in $24(96 \%)$ in the EUSr group and $20(80 \%)$ of those in the PTC grou Five patients in the EUSr and 5 patients in the PBD group underwent a pancreaticoduodenectomy at a median of 12 days (range 7 -165) following biliary drainage; there were no intraoperative of postoperative biliary complications.
EUSr was unsuccessful in 6 cases (24\%); summarized in $\bullet$ Table 3. Reasons for failed EUSr included inability to pass the guidewire antegrade into duodenum $(n=5)$, inability to locate a safe window EUS for biliary puncture due to duodenal deformity $(n=1)$. In cases where EUSr was unsuccessful, biliary drainage was obtained by PBD $(n=3)$, repeat ERCP $(n=2)$, and EUS-guided choledochoduodenostomy $(n=1)$. Both patients who had biliary access obtained through repeat ERCP underwent the procedure within 24 hours after EUSr was attempted. The median time to PBD following failed ERCP was 1 (range $0-3$ ) day. The median hospital length of stay for failed EUSr was 4 days (range $0-26$ ), which was similar to the primary PBD group (5 days, $P=0.64$ ).

\begin{tabular}{|c|c|c|c|}
\hline & $\begin{array}{l}\text { EUSr } \\
(n=25)\end{array}$ & $\begin{array}{l}\text { PBD } \\
(n=25)\end{array}$ & $P$ value \\
\hline Age (years; mean $\pm S D$ ) & $65.4 \pm 11.6$ & $67.7 \pm 13.6$ & 0.25 \\
\hline Gender (female) & 15 & 13 & 0.57 \\
\hline \multicolumn{4}{|l|}{ Etiology of biliary obstruction } \\
\hline Pancreatobiliary malignancy & 24 & 20 & 0.12 \\
\hline Metastatic malignancy & 1 & 5 & \\
\hline $\begin{array}{l}\text { Unsuccessful ERCP attempts } \\
\text { (median, range) }\end{array}$ & $1(1-3)$ & $1(1-2)$ & 0.26 \\
\hline \multicolumn{4}{|l|}{ Reason for failed ERCP } \\
\hline Tumor invasion/ deformity & 18 & 7 & 0.54 \\
\hline Normal ampulla & 16 & 9 & \\
\hline
\end{tabular}


Table 2 Post-procedure, per protocol analysis.

\begin{tabular}{|llll} 
& EUSr (n=25) & PBD (n=25) & P value \\
\hline Initial technical success & 19 & 25 & 0.009 \\
\hline Clinical success (reduction in bilirubin $>50 \%$ & $24 / 25(96 \%)$ & $20 / 25(80 \%)$ & 13.24 \\
\hline Mean pre-EUS-BD bilirubin & 10.85 & 3.13 & 0.022 \\
\hline Mean post-EUS-BD bilirubin & 1.89 & 5 days $(1-20)$ & 0.013 \\
\hline Length of hospital stay following initial drainage (median, range) & 1 day $(0-26)$ & $11(1-272)$ & 0.001 \\
\hline Follow-up duration after initial drainage (median, range) & 37 days (5-476) & 15 & 0.001 \\
\hline Repeat procedure & 3 & $1(0-10)$ &
\end{tabular}

\begin{tabular}{|c|c|c|c|c|c|c|c|c|c|c|}
\hline Patient & Age & $\begin{array}{l}\text { Year of } \\
\text { Study }\end{array}$ & $\begin{array}{l}\text { Endoscopist } \\
(1-5)\end{array}$ & $\begin{array}{l}\text { Primary } \\
\text { cancer }\end{array}$ & $\begin{array}{l}\text { Reason } \\
\text { EUSr failed }\end{array}$ & $\begin{array}{l}\text { Method of } \\
\text { biliary decom- } \\
\text { pression }\end{array}$ & $\begin{array}{l}\text { Length of } \\
\text { hospital } \\
\text { stay }\end{array}$ & $\begin{array}{l}\text { Clinical } \\
\text { success }\end{array}$ & $\begin{array}{l}\text { Early adverse } \\
\text { event(s) }\end{array}$ & $\begin{array}{l}\text { Repeat } \\
\text { biliary } \\
\text { intervention }\end{array}$ \\
\hline 1 & 69 & 2013 & 1 & Pancreatic & $\begin{array}{l}\text { Wire did } \\
\text { not pass } \\
\text { into } \\
\text { duodenum }\end{array}$ & PBD & 4 days & Yes & $\begin{array}{l}\text { Bleeding from } \\
\text { PBD tract, treat- } \\
\text { ed with PBD } \\
\text { replacement }\end{array}$ & yes \\
\hline 2 & 72 & 2013 & 1 & Pancreatic & $\begin{array}{l}\text { Could not } \\
\text { access bile } \\
\text { duct by EUS } \\
\text { due to } \\
\text { duodenal } \\
\text { deformity }\end{array}$ & ERCP & 4 days & Yes & None & yes \\
\hline 3 & 59 & 2013 & 1 & Ampullary & $\begin{array}{l}\text { Wire did } \\
\text { not pass } \\
\text { into } \\
\text { duodenum }\end{array}$ & EUS-BD & 26 days * & yes & None & no \\
\hline 4 & 75 & 2009 & 2 & Pancreatic & $\begin{array}{l}\text { Wire did } \\
\text { not pass } \\
\text { into } \\
\text { duodenum }\end{array}$ & PBD & 2 days & yes & None & no \\
\hline 5 & 49 & 2013 & 3 & Breast & $\begin{array}{l}\text { Wire did } \\
\text { not pass } \\
\text { into } \\
\text { duodenum }\end{array}$ & ERCP & 0 days & Yes & None & no \\
\hline 6 & 47 & 2014 & 1 & Pancreatic & $\begin{array}{l}\text { Wire did } \\
\text { not pass } \\
\text { into } \\
\text { duodenum }\end{array}$ & PBD & 7 days & Yes & None & no \\
\hline
\end{tabular}

EUSr, endoscopic ultrasound-guided rendezvous

Early AEs occurred in 4 (16\%) patients in the EUSr group and 3 $(12 \%)$ in the PBD groups $(P=0.68, \odot$ Table 3$)$. Early AEs included recurrent biliary obstruction (2 PBD), bleeding (2 EUSr, $1 \mathrm{PBD}$ ), pancreatitis (1 EUSr), and bile leak (1 EUSr). In the EUSr group, bleeding was related to a gastroduodenal artery pseudoaneurysm in 1 patient and was successfully treated with percutaneous embolization; a second patient developed hemobilia from a PBD after a failed EUSr, this was successfully treated by re-positioning of the PBD. In the PBD group one patient developed hemobilia; this was successfully treated with PBD repositioning and did not require vascular intervention. Delayed AE occurred in $3(12 \%)$ patients in the EUSr group and $6(5 \%)$ in the PBD group $(P=0.27)$ (৫ Table 4).

Repeat biliary intervention was required for 3 patients in the EUSr group and 14 in the PBD group $(P=0.001)$. Scheduled biliary interventions, including drain and stent changes were required in 14 patients in the PBD group, a median of 19 days (range 133) following initial drainage and in 3 patients in the EUSr group, a median of 5 days (range $1-105)$ later $(P<0.001)$. Conversion of PBD to metal biliary stent was successful in $10(40 \%)$ patients following initial PBD placement. Recurrent biliary obstruction, requiring intervention occurred in 4 patients in the PBD group, at a median of 237 days (range 1-272) after initial drainage and in one patient in the EUSr group, 476 days after stent placement (৫ Fig. 2, Log Rank $P=0.01$ ).

\section{Discussion \\ $\nabla$}

Since being first reported in 2001 by Giovannini et al., EUS-BD has been increasingly used as an alternative in patients with MDBO who fail ERCP [8]. Numerous techniques have been and are being developed with growing data supporting its use and efficacy $[1-5,9-14]$. In our study, EUSr was found to be a safe and effective method of biliary drainage in patients with MDBO after failed initial ERC It was associated with shorter post-procedure 
Table4 Adverse events.

\begin{tabular}{|c|c|c|c|}
\hline Adverse events & EUSr & PBD & \\
\hline Early (<7 days) & 4 & 3 & 0.68 \\
\hline Biliary obstruction & 0 & 2 & \\
\hline Bleeding & 2 & 1 & \\
\hline Pancreatitis & 1 & 0 & \\
\hline Bile leak & 1 & 0 & \\
\hline Late (>7 days) & 3 & 6 & 0.27 \\
\hline Biliary obstruction & 2 & 5 & \\
\hline Cholecystitis & 1 & 1 & \\
\hline
\end{tabular}

EUSr, endoscopic ultrasound-guided rendezvous; PBD, percutaneous biliary drainage

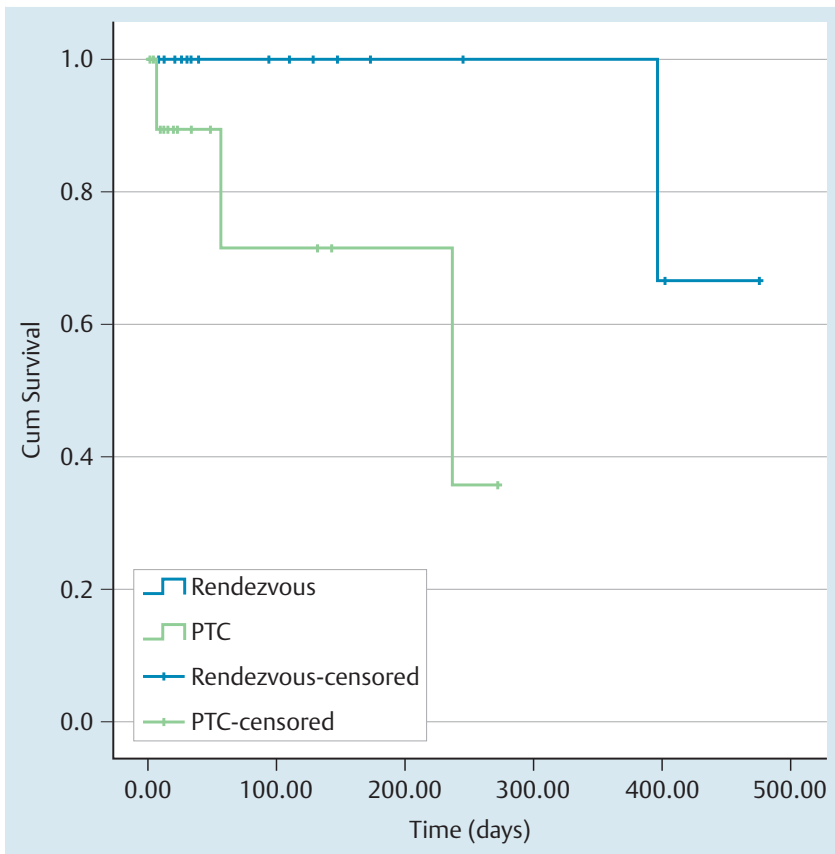

Fig. 2 Kaplan Meyer Estimate of time to biliary obstruction.

hospital stay and required fewer follow-up biliary interventions than PBD.

Three techniques have been developed including EUS-guided antegrade transluminal drainage (stenting using a transgastric or transduodenal approach without accessing the papilla), EUSguided antegrade transpapillary stent placement, and EUSr. From a large analysis of EUS-guided biliary techniques, the overall success rate of EUSr is estimated to be $81 \%$ with a complication rate about $10 \%$ of cases [6]. We recognize that there has been a more recent push for utilizing a protocol based approach to different rendezvous techniques, with some endoscopists favoring step wise attempts at biliary access starting with the trans-intrahepatic approach [15]. However, our standard approach to EUS-guided biliary access during the study period was EUSr, specifically utilizing the transduodenal approach with the echoendoscope seated in the duodenal bulb. This decision was based primarily on the concern for occurrence of bile leak, peritonitis and bleeding associated with creation of a fistula between the lumen of the gastrointestinal tract and the biliary tree when the antegrade approach is used or transluminal stenting is performed [6]. While these complications are relatively uncommon, there consequences can be devastating; particularly in the $20 \%$ of patients with potentially resectable pancreaticobiliary tumors.
With the recent introduction of lumen opposing stents, EUSguided choledochoduodenosotmy has become an attractive alternative to EUS-guided biliary decompression.[16] However, the currently available lumen opposing stents were not designed specifically for the biliary tree and may pose a risk of cholecystitis in patients with a gallbladder in situ as well as stent erosion or migration. Thus, we feel that if EUS guided biliary drainage is attempted rendezvous should remain the preferred first line approach, given the fact that it preserves the anatomic integrity of the biliary tree and avoid creation of a fistula.

It remains unclear whether EUS techniques are superior in efficacy, safety, and cost when compared to PBD in patients with MDBO who have failed ERC It is well known that PBD is highly successful but generally requires repeat procedures and periprocedural adverse events are not infrequent rates ranging from $10 \%$ to $32 \%$ in the modern literature [6]. In a recent study, Khashab et al studied a group of 73 patients with MDBO who failed repeat ERCP and found that technical success was higher in the PBD group but the adverse event rate was higher in the latter group of patients. Regarding successful biliary drainage, defined as reduction in total bilirubin by $50 \%$ in 2 weeks, the groups were not statistically different [2]. However, a major critique of the study is patients were enrolled to the PBD group only after failed ERCP and EUS-BD; potentially biasing the study against PBD.

In our study AE rates were similar between the EUSr and PBD groups. However, recurrent biliary obstruction occurred more frequently and repeated procedures were required more often in the PBD grou Repeated biliary manipulation may lead to further complications and requires the patient to have repeated hospital visits for catheter checks and changes. Recurrent biliary obstruction is a major source of morbidity and treatment interruption in patients undergoing chemotherapy. Given the fact that EUSr is associated with a lower rate of recurrent biliary obstruction it stands to reason that this approach would be preferable in patients receiving neoadjunct or palliative chemotherapy [17, 18]. Moreover, the length of hospital stay was significantly shorter in the EUSr group, landing another advantage to EUSr; particularly in cases where the initial ERCP is performed on an outpatient basis and if EUSr is performed in the same endoscopic session. Finally, we hypothesize that patients would experience a lower quality of life following PBD when compared to EUSr as external drainage catheters typically repeated interventions.

It cannot be understated however, that there are important advantages of PBD. First, PBD is more widely available and the techniques of $\mathrm{PBD}$ are well established and standardized. Furthermore, complications of PBD are typically minor and do not require surgical backup that may only be available at tertiary medical centers. That being said, EUS techniques have a key advantage of being performed at the same session of ERC However, if initial EUSr fails, it could be argued that PBD is a safer therapeutic option given no need for repeat anesthesia and its almost universal technical success. Technology, ie stents and guidewires, that focus solely on EUS-BD are needed and currently in development $[19,20]$. With the development of technology and as experience by interventional endoscopists is gained, the technical success rates of EUS-BD are likely to improve.

The major limitation of our study is its retrospective nature and limitations inherent to this design. The use of historical controls may have introduced some degree of bias; however there were no significant differences in the baseline characteristics and the protocol (minimum of 23 hours inpatient observation) following PBD placement remains the same at our institution. We also de- 
tected no difference in the rate of adverse events comparing EUSr and PBD, however our sample size may be insufficient to detect a meaningful difference in this regard. Additionally, this was a single-center study; results may not be applicable to institutions with different practice patterns for endoscopic and percutaneous biliary drainage. Finally, we focused our study on patients with malignant distal biliary obstruction and we cannot comment on the efficacy and safety of EUS guided biliary drainage in the treatment of hilar biliary structures or benign biliary disease.

\section{Conclusions}

In summary, EUSr is a safe and effective method of biliary drainage in patients with MDBO after failed ERC Initial technical success with EUSr was significantly lower than with PBD, however when EUSr was successful patients had a significantly shorter post-procedure hospital stay and required fewer follow-up biliary interventions than those undergoing PBD.

\section{Competing interests: None}

\section{References}

1 Sarkaria S, Lee HS, Gaidhane M et al. Advances in endoscopic ultrasound-guided biliary drainage: a comprehensive review. Gut Liver 2013; 7: 129-136

2 Khashab MA, Valeshabad AK, Afghani E et al. A comparative evaluation of EUS-guided biliary drainage and percutaneous Drainage in patients with distal malignant biliary obstruction and failed ERC. Dig Dis Sci 2015; 60: 557-565

3 Iwashita T, Lee JG, Shinovia S et al. Endoscopic ultrasound-guided rendezvous for biliary access after failed cannulation. Endoscopy 2012; 44: $60-65$

4 Artifon EL, Aparicio D, Paione JB et al. Biliary drainage in patients with unresectable, malignant obstruction where ERCP fails: endoscopic ultrasonography-guided choledochoduodenostomy versus percutaneous drainage. J Clin Gastroenterol 2012; 46: 768 -774

5 Hara K, Yamao K, Mizuno N et al. Endoscopic ultrasound-guided choledochoduodenostomy. Dig Endosc 2010; 22: 147-150

6 Iwashita T, Doi S, Yasuda I. Endoscopic ultrasound-guided biliary drainage: a review. Clin J Gastroenterol 2014; 7: 94-102
7 Covey AM, Brown KT. Percutaneous transhepatic biliary drainage. Tech Vasc Interv Radiol 2008; 11: 14-20

8 Giovannini M, Moutardier V, Pesenti C et al. Endoscopic ultrasoundguided bilioduodenal anastomosis: a new technique for biliary drainage. Endoscopy 2001; 33: 898-900

9 Gupta K, Mallery S, Hunter D et al. Endoscopic ultrasound and percutaneous access for endoscopic biliary and pancreatic drainage after initially failed ERC. Rev Gastroenterol Disord 2007; 7: 22 - 37

10 Khashab MA, Dewitt J. EUS-guided biliary drainage: is it ready for prime time? Yes! Gastrointest Endosc 2013; 78: 102-105

11 Mallery S, Matlock J, Freeman ML. EUS-guided rendezvous drainage of obstructed biliary and pancreatic ducts: Report of 6 cases. Gastrointest Endosc 2004; 59: 100 - 107

12 Iwashita T, Lee JG. Endoscopic ultrasonography-guided biliary drainage: rendezvous technique. Gastrointest Endosc Clin N Am 2012; 22: $249-258$

13 Liu YD, Wang ZQ Wang XD et al. Stent implantation through rendezvous technique of PTBD and ERCP: the treatment of obstructive jaundice. J Dig Dis 2007; 8: 198-202

14 Barkay O, Sherman S, McHenry L et al. Therapeutic EUS-assisted endoscopic retrograde pancreatography after failed pancreatic duct cannulation at ERC. Gastrointest Endosc 2010; 71: 1166-1173

15 Park do H, Jeong SU et al. Prospective evaluation of a treatment algorithm with enhanced guidewire manipulation protocol for EUS-guided biliary drainage after failed ERCP (with video). Gastrointest Endosc 2013; 78: $91-101$

16 Itoi $T$, Binmoeller $K$, Itowkawa $F$ et al. Endoscopic ultrasonographyguided cholecystogastrostomy using a lumen-apposing metal stent as an alternative to extrahepatic bile duct drainage in pancreatic cancer with duodenal invasion. Dig Endosc 2013; 25: 02137-141

17 Boulay BR, Gardner TB, Gordon SR. Occlusion rate and complications of plastic biliary stent placement in patients undergoing neoadjuvant chemoradiotherapy for pancreatic cancer with malignant biliary obstruction. J Clin Gastroenterol 2010; 44: 452-455

18 Sut M, Kennedy R, McNamee J et al. Long-term results of percutaneous transhepatic cholangiographic drainage for palliation of malignant biliary obstruction. J Palliat Med 2010; 13: 1311-1313

19 Itoi T, Binmoeller KF, Shah J et al. Clinical evaluation of a novel lumenapposing metal stent for endosonography-guided pancreatic pseudocyst and gallbladder drainage (with videos). Gastrointest Endosc 2012; 75: $870-876$

20 Itoi $T$, Itokawa $F$, Uraoka $T$ et al. Novel EUS-guided gastrojejunostomy technique using a new double-balloon enteric tube and lumen-apposing metal stent (with videos). Gastrointest Endosc 2013; 78: 934-939 